\title{
Hidrododinâmica do solo em uma área degradada na região do Cariri Paraibano
}

\author{
Soil hydrododynamics in a degraded area in the Cariri region of Paraíba \\ Hidrodinámica del suelo en una zona degradada de la región Cariri de Paraíba
}

Recebido: 19/12/2021 | Revisado: 27/12/2021 | Aceito: 29/12/2021 | Publicado: 06/01/2022

\author{
Vitória Dantas Sousa \\ ORCID: https://orcid.org/0000-0003-4801-886X \\ Universidade Federal de Campina Grande, Brasil \\ E-mail: vitória.dantas@estudante.ufcg.edu.br \\ Maria Sallydelândia Sobral de Farias \\ ORCID: https://orcid.org/0000-0002-3255-2024 \\ Universidade Federal de Campina Grande, Brasil \\ E-mail: sallyfarias@hotmail.com \\ Jean Pereira Guimarães \\ ORCID: https://orcid.org/0000-0002-2615-9832 \\ Universidade Federal de Campina Grande, Brasil \\ E-mail:1p_jean@hotmail.com \\ Júlia Soares Pereira \\ ORCID: https://orcid.org/0000-0002-6495-1169 \\ Universidade Federal de Campina Grande, Brasil \\ E-mail: julia_eng@hotmail.com
}

\begin{abstract}
Resumo
Da caracterização da hidrodinâmica do solo, surgi subsídios para um manejo adequado da área seja de sequeiro ou irrigada em regiões semiáridas estudos nesta linha de pesquisa tem sido muito escasso e que vem levando muitas áreas ao processo de degradação comprometendo e produção. Objetivou-se com esta pesquisa avaliar os parâmetros, hidrodinâmicos do solo, além de identificar a possível necessidade de drenagem do solo em uma área em recuperação ambiental. A área experimental está localizada no município de São José dos Cordeiros - PB, localizado no Cariri Paraibano. Foram realizadas dez amostras de solos para avaliação dos parâmetros físicos na $30 \mathrm{~cm}$ de profundidades. As análises foram realizadas no laboratório de irrigação e Salinidade. A condutividade hidráulica saturada (K0) pelo método de Auger Hole inverso (Porchet), realizadas em campo. O solo apresenta uma textura franco, franco argiloarenoso e franco arenoso, os valores de densidade do solo variaram de 1,30 a $1,61 \mathrm{~g} \mathrm{~cm}^{-3}$, para densidade da partícula a variação foi 2,68 a $2,72 \mathrm{~g} \mathrm{~cm}^{-3}$.A condutividade hidráulica saturada do solo variou de 0,39 a 1,5 m.dia ${ }^{-1}$ na área, sendo classificada como lenta e moderada. A porosidade drenável variou de 31,28 a 42,79.
\end{abstract}

Palavras-chave: Solo; Degradação; Física do solo.

\begin{abstract}
From the characterization of the soil hydrodynamics, there are subsidies for an adequate management of the area, whether rainfed or irrigated in semiarid regions, studies in this line of research have been very scarce and have been leading many areas to the degradation process, compromising production. The objective of this research was to evaluate the hydrodynamic parameters of the soil, in addition to identifying the possible need for soil drainage in an area undergoing environmental recovery. The experimental area is located in the municipality of São José dos Cordeiros - PB located in Cariri Paraibano. Ten soil samples were taken to evaluate the physical parameters at a depth of $30 \mathrm{~cm}$. Analyzes were performed in the irrigation and salinity laboratory. The saturated hydraulic conductivity (K0) by the inverse Auger Hole method (Porchet), performed in the field. The soil has a loam, sandy clay loam and sandy loam texture, the soil density values ranged from 1.30 to $1.61 \mathrm{~g} \mathrm{~cm}^{-3}$, for particle density the variation was 2.68 to $2.72 \mathrm{~g} \mathrm{~cm}^{-3}$. The saturated hydraulic conductivity of the soil ranged from 0.39 to $1.5 \mathrm{~m} . \mathrm{day}^{-1}$ in the area, being classified as slow and moderate. The drainable porosity ranged from 31.28 to 42.79 .
\end{abstract}

Keywords: Soil; Degradation; Soil physics.

\section{Resumen}

A partir de la caracterización de la hidrodinámica del suelo, existen subsidios para un adecuado manejo de la zona, ya sea de secano o de regadío en regiones semiáridas, los estudios en esta línea de investigación han sido muy escasos y han llevado muchas áreas al proceso de degradación, comprometiendo la producción. El objetivo de esta investigación fue evaluar los parámetros hidrodinámicos del suelo, además de identificar la posible necesidad de drenaje del suelo en un área en recuperación ambiental. El área experimental está ubicada en el municipio de São José dos Cordeiros PB ubicado en Cariri Paraibano. Se tomaron diez muestras de suelo para evaluar los parámetros físicos a una 
profundidad de $30 \mathrm{~cm}$. Los análisis se realizaron en el laboratorio de riego y salinidad. La conductividad hidráulica saturada (K0) por el método inverso del barreno (Porchet), realizado en campo. El suelo tiene una textura franco, franco arcillosa arenosa y franco arenosa, los valores de densidad del suelo variaron de 1.30 a $1.61 \mathrm{~g} \mathrm{~cm}^{-3}$, para la densidad de partículas la variación fue de 2.68 a $2.72 \mathrm{~g} \mathrm{~cm}^{-3}$. La conductividad hidráulica saturada del El suelo osciló entre 0,39 y 1,5 m.día ${ }^{-1}$ en la zona, clasificándose como lento y moderado. La porosidad drenable osciló entre 31,28 y 42,79.

Palabras clave: Suelo; Degradación; Física del suelo.

\section{Introdução}

Nas regiões áridas e semiáridas a salinização do solo constitui um sério problema, limitando a produção agrícola e reduzindo a produtividade das culturas. Em condições normais essas regiões apresentam altas temperaturas e baixos índices pluviométricos distribuídos de forma irregular no tempo e no espaço. O manejo inadequado da irrigação, a qualidade da água e as condições de drenagem insuficiente, contribuem para a aceleração do processo de salinização do solo. Portanto, a prática de irrigação deve ser usada de forma racional uma vez que as condições de clima do Nordeste e os elevados teores de sais nas águas de irrigação, têm causado salinização dos solos (Figueiredo et al., 2009). É caracterizado pela precipitação pluviométrica média em torno de $800 \mathrm{~mm}$ anuais, índice de aridez de Thornthwaite igual ou inferior a 0,50, e déficit hídrico diário igual ou superior a 60\% (SUDENE, 2017). As variações espaciais e temporais nas condições climáticas, juntamente com a diversidade de rochas $(70 \%$ cristalina e $30 \%$ sedimentares) e o relevo, condicionam a grande diversidade de solos e formações vegetais no semiárido brasileiro (Marques et al., 2014). Em decorrência dessa heterogeneidade nas condições abióticas é comum encontrar solos arenosos e profundos a poucos metros de distância de solos argilosos e rasos, e diferentes formações vegetais associadas.

A gestão eficiente dos recursos hídricos é uma das principais estratégias para o desenvolvimento sustentável nas áreas com escassez hídrica. O gerenciamento integrado contribui para evitar o desperdício e reduzir a incidência de conflitos do uso da água. Deve-se buscar, portanto, um cenário do uso racional deste recurso, procurando um equilíbrio entre as demandas da sociedade e as disponibilidades efetivas das águas superficiais e subterrâneas (Medeiros et. al., 2011).

O estudo da hidrodinâmica dos solos em regiões semiáridas é de fundamental importância, pois existe uma carência de informações na região direcionada a dinâmica de água no solo em áreas irrigadas ou de cerqueiro. O estudo deve começar por uma avaliação do solo principalmente nas características físicas. A condutividade hidráulica saturada do solo (K0) representa um parâmetro chave para análise da intensidade do deslocamento da água no solo. Para a sua determinação existe uma grande variedade de métodos, com diferentes níveis de precisão; alguns aplicáveis apenas em determinadas condições ou com certos objetivos, os quais, de modo geral, podem ser agrupados em métodos indiretos ou diretos (Marques et. al. 2008).

No campo, destacam-se os métodos que usam o princípio do fluxo contínuo, como o furo de trado (poço), os de fluxo constante, sendo que o princípio do fluxo contínuo é mais utilizado; os mais aplicados são o do furo de trado (não aconselhado para solos arenosos) e o método de Porchet, também conhecido como método inverso, uma vez que mede a K(0) na ausência do lençol freático (Barreto,2001).

Pesquisa de Guimarães (2017), em uma área da mesma propriedades onde está sendo realizada a pesquisa em curso, encontrou valores de condutividade hidráulica saturada do solo que variou entre 0,10 a 0,58 m.dia-1, e a porosidade drenável variando entre 27,72 a $42,46 \%$, valores considerados de alta variabilidade a muito alta, enquanto que a $\mu$ obteve baixa e média variabilidade.

Baseado nesta explanação o objetivo geral deste trabalho é realizar uma caracterização da hidrodinâmica do solo em uma área degradada no Cariri Paraibano. E os objetivos específicos são analisar o solo nos parâmetros físicos; determinar a porosidade drenável e a condutividade hidráulica em campo. 
Research, Society and Development, v. 11, n. 1, e27311124937, 2022

(CC BY 4.0) | ISSN 2525-3409 | DOI: http://dx.doi.org/10.33448/rsd-v11i1.24937

\section{Metodologia}

O município de São José do Cordeiros (Figura 01) situa-se no Polígono da Secas. Possui clima Bsh semiárido quente com chuvas de verão. A pluviometria média anual é de 554,5mm de distribuição irregular, com $78 \%$ concentrada em quatro meses. A vegetação é do tipo Caatinga-29 Sertão e a temperatura média anual próximo a $24{ }^{\circ} \mathrm{C}$. A topografia apresenta relevo ondulado à suavemente ondulado (Mascarenhas, 2005).

Figura 1: Área experimental localizada no município de São José dos Cordeiros - Paraíba.

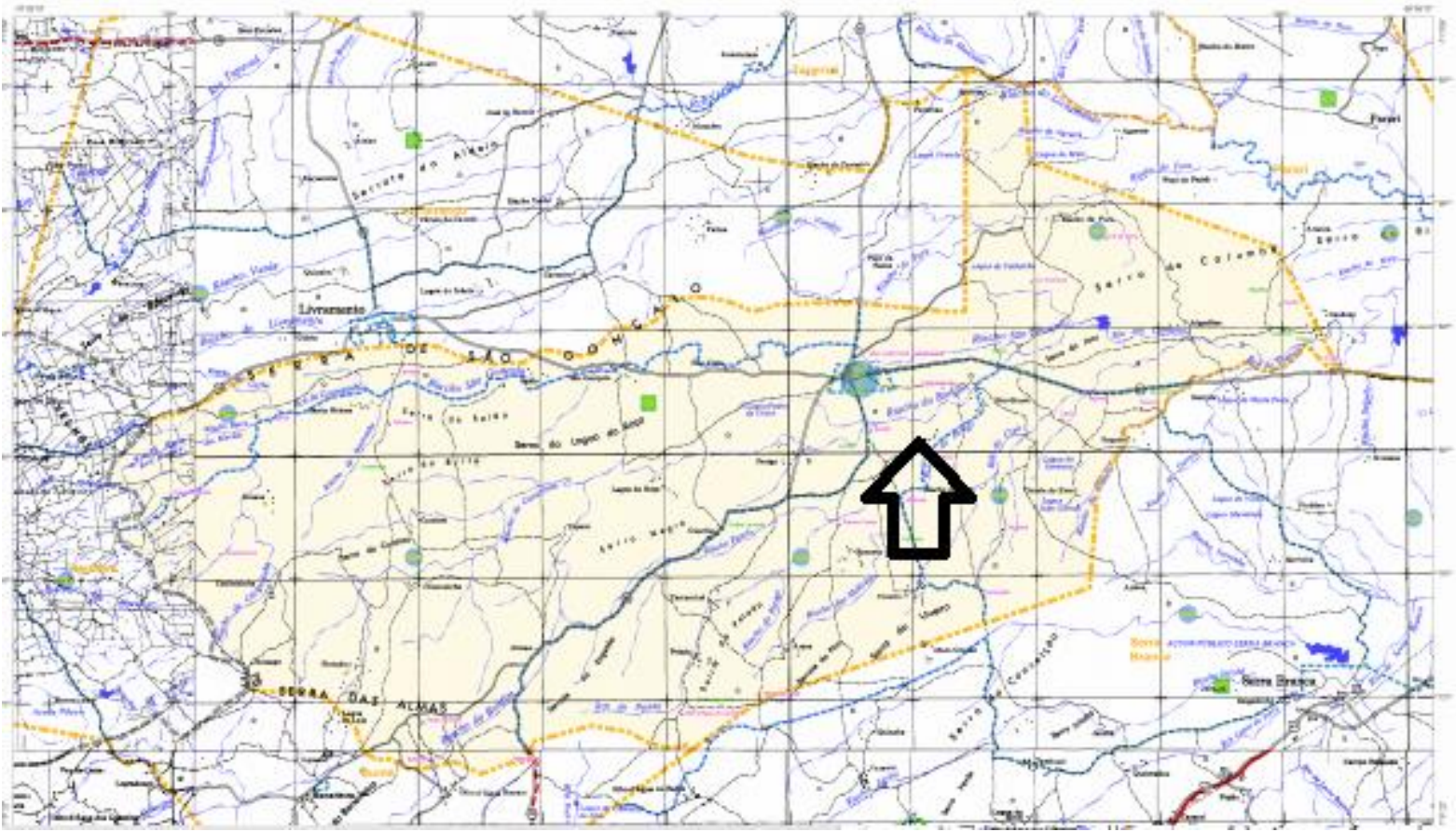

Fonte: IBGE (2021).

As declividades mais elevadas onde predomina o relevo ondulado apresentam-se à noroeste onde ocorre a serra de São Gonçalo com cotas que chegam a 670 metros; na porção sudoeste onde ocorre a serra das Almas com cotas que chegam a 780 metros, sul, nas áreas onde ocorrem as serras do Viveiro, da Pelada e Serrote do Pico e, a oeste em áreas separando os altos cursos dos riachos São Gonçalo e do Bonfim onde ocorrem as serras do Salão e da Roca com cotas que chegam a 670 metros. A vegetação é do tipo Caatinga e a temperatura média situa-se entre torno de $24^{\circ} \mathrm{C}$. (MASCARENHAS, 2005).

A área experimental de desenvolvimento da pesquisa é no Sitio do Meio. Apresenta uma extensão de 20 ha, onde 2 hectares encontra-se em processo de recuperação com atividades desenvolvidas pelo projeto de recuperação implantado na área em 2016 (CAPITULINO, 2019). Foram coletadas 10 amostras do solo para avaliação física, a profundidade de coleta foi de 0$30 \mathrm{~cm}$. As análises foram realizadas no Laboratório de Irrigação e Salinidade (LIS) - UFCG segundo metodologia da (EMBRAPA,1997).

A porosidade drenável $(\mu)$, também conhecida como porosidade efetiva ou macroporosidade, é considerada a fração da porosidade total através da qual a água move-se livremente, cujo valor equivale ao conteúdo de ar presente no solo na capacidade de campo. Para este estudo, os valores de porosidade drenável foram calculados seguindo a equação proposta por Beltran (1986):

Equação 01: 
$\mu=P T-U U_{C C}$

Em que: $\mu$ - Porosidade drenável (\%); PT - Porosidade total (\%); Ucc - Umidade do solo na capacidade de campo.

Foi realizado uma determinação da condutividade hidráulica em campo o método inverso de Auger-Hole, também conhecido como o método de "Porchet", indicado para áreas em que o lençol freático encontra-se em grandes profundidades ou ausente, que consiste em abrir um orifício no solo, de certa profundidade, enchê-lo de água e medir a velocidade de rebaixamento do nível. Os cálculos da condutividade hidráulica (K0) seguem a seguinte equação (MILLAR, 1988):

Equação 02:

$$
K=1,15 r \frac{\log y_{1}+\frac{r}{2}-\log y_{2}+\frac{r}{2}}{t_{2}-t_{1}}
$$

Onde: $\mathrm{K} 0=$ condutividade hidráulica $\left(\mathrm{m} \mathrm{dia}^{-1}\right) ; \mathrm{h} 0=$ carga hidráulica no instante "to" $(\mathrm{cm}) ; \mathrm{hf}=$ carga hidráulica no instante " $t \mathrm{f}$ " (cm); r = raio de perfuração $(\mathrm{cm}) ; \mathrm{t} 0=$ Tempo inicial (s); $\mathrm{tf}=$ Tempo final $(\mathrm{s}) ; \mathrm{h} 0=$ Leitura inicial - Profundidade do poço - Altura do plano de referência; hf = Leitura final - Profundidade do poço - Altura do plano de referência. Figura 2.

Os modelos matemáticos são ferramentas eficientes na simulação de mudanças físicas, químicas e biológicas em sistemas aquáticos. Contudo, é necessário que as informações de saída sejam corretamente interpretadas para que ações corretivas possam ter impactos positivos (SONG; KIM, 2009).

$\mathrm{Na}$ realização dos testes de condutividade em campo foram utilizados os seguintes materiais: ferramentas para a limpeza da área, conjunto de trado tipo holandês com diâmetro de perfuração de 7,5 cm e alcance de profundidade de $1,5 \mathrm{~m}$ para abertura dos poços, baldes para coleta e armazenamento da água utilizada, suporte para medir a variação de nível de água dentro do poço, trena de $2 \mathrm{~m}$ acoplada a um flutuador para a medição do nível de água no poço. Os testes de condutividade hidráulica foram realizados em dez poços (Figura 3) distribuídos aleatoriamente na área de estudo com uma profundidade de 1,5 metros. Foram realizados 3 testes por poço e retirado o valor médio.

Figuras 2 e 3: Poços de observação sendo medido a condutividade hidráulica e poço instalado.

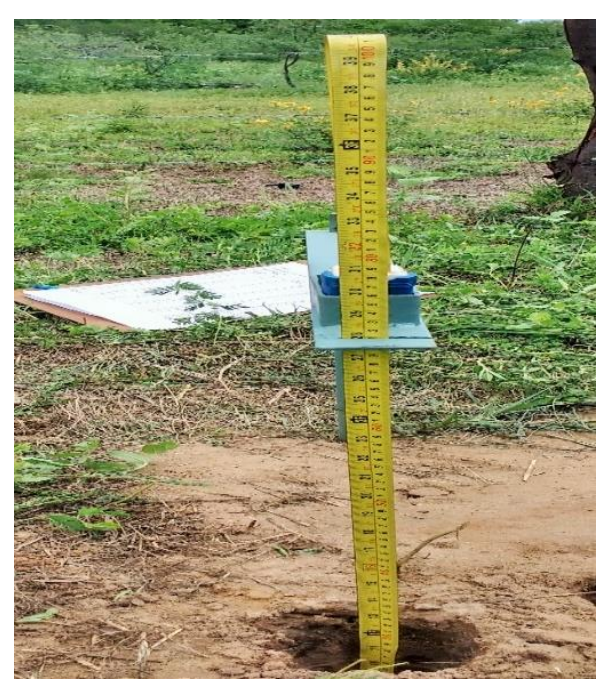

Fonte: Autores.

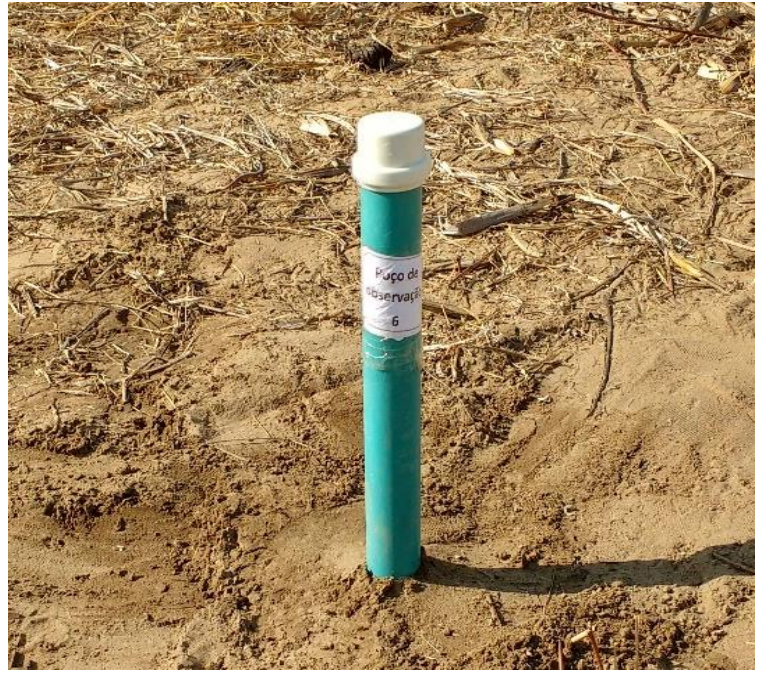

Fonte: Autores 


\section{Resultados e Discussão}

Com base nos resultados da análise física das amostras de solos, observou-se as seguintes classes texturais: franco, franco argilo-arenoso e franco arenoso. Através dos dados de analises de solo observou-se que o solo tem características arenosa na profundidade avaliada.

Os valores de densidade do solo variaram de 1,30 a 1,61 $\mathrm{g} \mathrm{cm}^{-3}$, para densidade da partícula a variação foi 2,68 a 2,72 $\mathrm{g} \mathrm{cm}^{-3}$, a porosidade variou de 40,15 a 52,21\%. Guimarães (2017) em pesquisa de Diagnóstico de Drenagem na área estudada, encontrou valores de teores de areia se sobressaíram aos de silte e argila para todas as profundidades analisadas, sendo as maiores médias encontradas nas profundidades de 0 a $30 \mathrm{~cm}$ para a areia $(85,90 \%)$, e 90 a $120 \mathrm{~cm}$ para o silte $(16,66 \%)$ e a argila $(9,12 \%)$, dados que corroboram com os da pesquisa.

A porosidade drenável variou de 31,28 a 42,79\%. Segundo Silva \& Kato (1997), a macroporosidade é fator de extrema importância na condutividade hidráulica do solo saturado e sua redução provoca diminuição nos valores de K0. Esses autores observaram que pequena diferença de macroporosidade entre áreas de manejo convencional e de plantio direto foi acompanhada por grande diferença nos valores de K0, sendo constatado o valor de 0,0035 cm s-1, na primeira, e o de 0,0231 cm s-1, na segunda, evidenciando a importância da macroporosidade na condutividade hidráulica, ou seja, o efeito do manejo na estrutura do solo.

De acordo com os resultados obtidos para as condutividades hidráulicas na área observou-se que houve uma variação de 0,39 a 1,5 m/dia, segundo Millar (1988), valores entre 0,1 a 0,5 cm h-1 é considerada lenta e valores entre 0,5-2,0 cm h-1, moderada. Existe na área problemas de compactação o dificulta o movimento da água no solo, fruto de processo de degradação da área. Guimarães (2017) encontrou valores na mesma propriedade Sitio do Meio de 0,10 a 0,58 m.dia-1, sendo na área de cultivo.

Segundo Borges et. al (2015), o conhecimento da condutividade hidráulica é fundamental para qualquer estudo que envolva o movimento da água no solo, o transporte de elementos químicos, nutrientes e defensivos agrícolas, bem como a descrição da funcionalidade de seu sistema poroso, no qual, engloba propriedades relacionadas com a sua porosidade, como quantidade, tamanho, morfologia, continuidade e orientação dos poros.

Souza e colaboradores (2014) estudando a hidrodinâmica do solo no cariri paraibano encontraram variação de valores de condutividade hidráulica do solo que variaram de 0,35 a 0,96 m/dia utilizando o método de Porchet (Inverso de AugerRole).

\section{Conclusão}

O solo apresenta uma textura franco, franco argilo-arenoso e franco arenoso;

Os valores de densidade do solo variam entre 1,30ea $1,61 \mathrm{~g} \mathrm{~cm}-3$, para densidade da partícula a variação de 2,68 a $2,72 \mathrm{~g} \mathrm{~cm}-3$;

A condutividade hidráulica saturada do solo variou de 0,39 a 1,5 m.dia-1, sendo classificada como lenta e moderada; A porosidade drenável variou entre 31,28 a 42,79 .

\section{Referências}

Barreto, A. N., Oliveira, G. R., Nogueira, L. C.; IVO, W. M. P. M. (2001). Condutividade hidráulica saturada em um solo aluvial do perímetro irrigado de São Gonçalo, PB. Revista Brasileira de Engenharia Agrícola e Ambiental, vol.5, $\mathrm{n}^{\circ} 1$.

Bernardo, R. S. (2005). Condutividade hidráulica de três solos da região norte fluminense. (Dissertação de Mestrado). Rio de Janeiro. 
Research, Society and Development, v. 11, n. 1, e27311124937, 2022

(CC BY 4.0) | ISSN 2525-3409 | DOI: http://dx.doi.org/10.33448/rsd-v11i1.24937

Borges, T. K. S.; Silva, N. D.; Cavalcante, A. R.; Coto, J. P. C. (2015). Estimativa da condutividade hidráulica pelo método furo de trado. IN: XXV CONIRD - Congresso Nacional de Irrigação e Drenagem, UFS - São Cristóvão/SE. 08 a 13 de novembro.

Capitulino, Jessica Dayanne. et al. (2019). Regulação De Fluxos Hídricos Em Área Degradada Por Erosão No Cariri Paraibano. In: SEABRA, Giovanni (org.). Terra - Mudanças Climáticas e Biodiversidade. Ituiutaba: Barvalento.

Cavalcante, L. F. (2000). Sais e seus problemas nos solos irrigados. Areia: UFPB.

Cruciani, D. E. (1940). A drenagem na agricultura. São Paulo, Nobel.

EMBRAPA (1997). Manual de métodos de análise de solo. 2 ed. Rio de Janeiro: EMBRAPA. Serviço Nacional de Levantamento e Conservação de Solo.

Felipe G. S.; Farias, M.S.S.; Silva, T. T.; Ferreira Filho, J. G.A.; Saboya, L.M.F. (2014). Hidrodinâmica do solo em uma área destinada à agricultura familiar no cariri paraibano. Revista Verde (Mossoró - RN - BRASIL), v. 9, n.1, p.13 -17.

Guimarães, J. P. (2017). Dinâmica da drenagem em solos da caatinga em uso com palma forrageira irrigada e culturas de subsistência. Dissertação de mestrado. UFCG.

Lima Júnior, B. C. (2013). Diagnóstico da necessidade de drenagem em solo cultivado com fruticultura irrigada com água poluída. Dissertação de Mestrado em Engenharia Agrícola. UFCG/CTRN. Campina Grande, PB.

Marques, F. A., Nascimento, A. F., Araujo Filho, J. C., Silva, A. B. 2014. Solos do Nordeste. Recife: Embrapa Solos.

Marques, J. D. O.; Teixeira, G. W; Reis, M. A.; Cruz Junior, O.F.; Martins, C. G. (2008). Avaliação da condutividade hidráulica do solo saturada utilizando dois métodos de laboratório numa topossequência com diferentes coberturas vegetais no Baixo Amazonas. Ver. ACTA Amazonica. vol. 38

Mascarenhas, J. C.; Beltrão, B. A.; Junior, L. C. S.; Morais, F.; Mendes, V. A.; Miranda, J. L. F. (2005). Projeto cadastro de fontes de abastecimento por água subterrânea. Diagnóstico do município de São José dos Cordeiros, estado da Paraíba. Recife: CPRM/PRODEEM.

Marques, J.D.O. Eixeira, W.G.; Resis, A. M.; Cruz Junior, O.F.; Martins, G. C. T. (2008). Avaliação da condutividade hidráulica do solo saturada utilizando dois métodos de laboratório numa topossequência com diferentes coberturas vegetais no Baixo Amazonas. ACTA Amazônica. vol. 38.

Medeiros, C. N.; Gomes, D M.; Albuquerque, E. L S, Cruz, M. L. B. (2011). Os Recursos Hídricos do Ceará: Integração, Gestão e Potencialidades. IPECE.

Millar, A. A. (1988). Drenagem de terras agrícolas: bases agronômicas. São Paulo, Editerra Editora Ltda.

Silva, C. L. \& Kato, E. (1997). Efeito do selamento superficial na condutividade hidráulica saturada da superfície de um solo sob cerrado. Pesq. Agropec. Bras., 32:213-220.

Song, Tao; KIM, Kyehyun. (2009). Development of a water quality loading index based on water quality modeling. Journal of Environmental Management. v.90, n.3, p. 1534-1543, março.

Souza, F. G.; Farias, M. S. S.; Silva, T. T. S.; Ferreira Filho, J. G. A.; Saboya, L. M. F. (2014). Hidrodinâmica do solo em uma área destinada à agricultura familiar no cariri paraibano. Revista Verde de Agroecologia e Desenvolvimento Sustentável. v. 9, n. 1, p. 13-27, Mossoró, RN.

Sudene. Superintendência de Desenvolvimento do Nordeste. 2017 Resolução nº107 de 27 de julho de 2017 < https://www.lex.com.br/legis_27508570_RES OLUCAO_N_107_DE_27_DE_JULHO_DE_2017 